DAMTP-R/97/10

QMW-PH-97-9

NI-970015

hep-th/9702202

\title{
Hyper-Kähler Manifolds and Multiply Intersecting Branes
}

\author{
J.P. Gauntlett ${ }^{\star}$ \\ Dept. of Physics, Queen Mary and Westfield College, \\ Mile End Rd., London E1 4NS, U.K. \\ and \\ G.W. Gibbons * , G. Papadopoulos and P.K. Townsend * \\ DAMTP, University of Cambridge, \\ Silver St., Cambridge CB3 9EW, U.K.
}

\begin{abstract}
Generalized membrane solutions of $\mathrm{D}=11$ supergravity, for which the transverse space is a toric hyper-Kähler manifold, are shown to have IIB duals representing the intersection of parallel 3-branes with 5-branes whose orientations are determined by their $S l(2 ; \mathbb{Z})$ charge vectors. These IIB solutions, which generically preserve $3 / 16$ of the supersymmetry, can be further mapped to solutions of $\mathrm{D}=11$ supergravity representing the intersection of parallel membranes with any number of fivebranes at arbitrary angles. Alternatively, a subclass (corresponding to nonsingular $\mathrm{D}=11$ solutions) can be mapped to solutions representing the intersection on a string of any number of D-5-branes at arbitrary angles, again preserving 3/16 supersymmetry, as we verify in a special case by a quaternionic extension of the analysis of Berkooz, Douglas and Leigh. We also use similar methods to find new $1 / 8$ supersymmetric solutions of orthogonally intersecting branes.
\end{abstract}

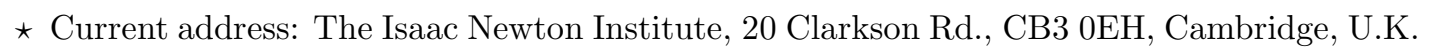




\section{Introduction}

A number of recent developments have underscored the importance of supersymmetric intersecting p-brane configurations of M-theory and superstring theories for a variety of phenomena of physical interest. Much of the work on this subject has been concerned with the realization of intersecting p-brane configurations as solutions of the relevant effective supergravity theory. The solutions so far considered have been restricted to those representing orthogonal intersections. Furthermore, most are 'delocalized' in some directions, with the consequence that their interpretation as intersecting branes is not completely straightforward. In this paper we report on some progress towards lifting these restrictions. A novelty of many of the new solutions we find is that they preserve 3/16 supersymmetry, a fraction not obtainable from orthogonal intersections. Our method derives from considering certain solutions of $\mathrm{D}=11$ supergravity so it has the additional advantage that an M-theory setting is automatically provided. Moreover, many of these $\mathrm{D}=11$ supergravity solutions are non-singular, thereby providing examples of how the singularities of at least some intersecting brane solutions of type II superstring theory are resolved in M-theory.

Using M-theory and superstring dualities, any intersecting brane solution can be obtained from some solution of $\mathrm{D}=11$ supergravity, although it does not follow from this fact that the $\mathrm{D}=11$ solution will also have an intersecting brane interpretation. Conversely, solutions of $\mathrm{D}=11$ supergravity with some other interpretation, or with no obvious interpretation at all, may be interpretable as intersecting brane solutions after reduction to $\mathrm{D}=10$ and possible dualization. If one regards a single brane as a degenerate case of an intersecting brane configuration then a case in point is the solution of $\mathrm{D}=11$ supergravity for which the 11-dimensional metric is the product of 7-dimensional Minkowski space with the (hyper-Kähler) Euclidean Taub-Nut metric. Since the latter metric admits a U(1) isometry and is asymptot-

ically flat, this solution can be reduced to $\mathrm{D}=10$ where it becomes the $\mathrm{D}$-6-brane solution of IIA supergravity [1]. Similarly, the analogous Euclidean Taub-Nut so- 
lution of $\mathrm{D}=10 \mathrm{IIA}(\mathrm{B})$ supergravity is T-dual to an NS-5-brane solution of IIB(A) supergravity [2].

This raises an obvious question: does the product of two Euclidean Taub-Nut spaces, which is an 8-dimensional asymptotically flat hyper-Kähler manifold with holonomy $S p(1) \times S p(1)$, have an analogous interpretation when used as a solution of $\mathrm{D}=11$ supergravity? The same question can also be asked of any 8-dimensional asymptotically flat hyper-Kähler manifold, for which the holonomy is generically $S p(2)$. One purpose of this paper is to provide an answer to this question for asymptotically flat 8-dimensional 'toric' hyper-Kähler manifolds, i.e. those admitting a triholomorphic $T^{2}$ isometry, by showing that the associated solutions of $\mathrm{D}=11$ supergravity are mapped to intersecting 5 -brane solutions of IIB supergravity. The generic IIB solution found this way preserves $3 / 16$ of the supersymmetry of the IIB vacuum solution and is naturally interpreted as an arbitrary number of 5 -branes with pairwise intersections at angles determined by their $S l(2 ; \mathbb{Z})$ charges.

The fact that these solutions generically preserve 3/16 supersymmetry derives in the first instance from the fact that hyper-Kähler 8-manifolds generically have holonomy $S p(2)$, because this implies the preservation of $3 / 16$ supersymmetry by the $\mathrm{D}=11$ supergravity solution; the triholomorphicity of the $T^{2}$ isometry then ensures that this feature is maintained under dimensional reduction to $D=10$ and subsequent T-duality. In the special case in which the 8-metric is the metric product of two 4-metrics the holonomy is reduced to $S p(1) \times S p(1)$ and the corresponding solutions of $\mathrm{D}=11$ supergravity, which preserve $1 / 4$ of the supersymmetry, are mapped under duality to solutions representing any number of parallel D-5-branes orthogonally intersecting, or overlapping, any number of parallel NS-5-branes on a 2-brane.

These IIB solutions can now be mapped back to $\mathrm{D}=11$ to give new intersecting M-5-brane solutions in which an arbitrary number of M-5-branes intersect at arbitrary angles while still preserving 3/16 supersymmetry. Alternatively, a series of duality transformations leads to a class of solutions of IIB supergravity repre- 
senting the intersection on a string of an arbitrary number of D-5-branes, again at arbitrary angles and preserving 3/16 of the supersymmetry. A potentially useful feature of these solutions is that, since they involve only D-branes, it should be possible to further analyse them using string perturbation theory. A special case represents just two D-5-branes intersecting on a string with one rotated by an arbitrary angle relative to the other. When the D-5-branes are orthogonal they preserve $1 / 4$ of the supersymmetry, as expected, so we learn from the more general solution that a rotation away from orthogonality may be such as to preserve $3 / 4$ of the original supersymmetry. One might have expected that any deviation from orthogonality would break all supersymmetries, but it has been shown previously by other methods that this is not necessarily the case [3,4]. We adapt these methods to our case to verify that the fraction of supersymmetry preserved, relative to the vacuum, is $3 / 16$.

The starting point for all the above results are non-singular solutions of $\mathrm{D}=11$ supergravity for which the $\mathrm{D}=11$-form field strength is zero and the 11-metric takes the form

$$
d s^{2}=d s^{2}\left(\mathbb{E}^{2,1}\right)+d s_{8}^{2}
$$

where $\mathbb{E}^{2,1}$ is $\mathrm{D}=3$ Minkowski space and $d s_{8}^{2}$ is a complete toric hyper-Kähler 8metric. This solution is actually a special case of a 'generalized membrane' solution for which

$$
\begin{aligned}
d s^{2} & =H^{-\frac{2}{3}} d s^{2}\left(\mathbb{E}^{2,1}\right)+H^{\frac{1}{3}} d s_{8}^{2} \\
F & = \pm \omega_{3} \wedge d H^{-1}
\end{aligned}
$$

where $\omega_{3}$ is the volume form on $\mathbb{E}^{2,1}$ and $H$ is a $T^{2}$-invariant ${ }^{\star}$ harmonic function on the hyper-Kähler 8-manifold. Provided the sign of the expression for the 4form $F$ in (1.2) is chosen appropriately, the solution with $F \neq 0$ breaks no more supersymmetries than the solution (1.1) with $F=0$.

* This condition on $H$ is needed for our applications; it is not needed to solve the $\mathrm{D}=11$ supergravity equations. Solutions of the form of (1.2) have been found previously in the context of KK theory (see [5] for an M-theory update). Generalized membrane solutions of a rather different type, but preserving $3 / 16$ supersymmetry, have also been found [6]. 
Since the 'generalized membrane' solution (1.2) of $\mathrm{D}=11$ supergravity admits the action of a torus we can convert it to a solution of IIB $\mathrm{D}=10$ supergravity, as in the pure hyper-Kähler case. The resulting IIB solution generalizes the previous one in that the 2-brane overlap of the 5-branes now has the interpretation as the intersection (or, possibly, the boundary) of a D-3-brane with the 5-branes. In the case of orthogonal intersections these are just the configurations used by Hanany and Witten (HW) in their study of $\mathrm{D}=3$ supersymmetric gauge theories [7]. Actually, our supergravity solutions do not quite correspond to the HW configurations because they are translationally invariant along the direction in the 3-brane connecting the 5-branes. In another respect, however, our solutions are more general because they include configurations of non-orthogonal 5-branes preserving 3/16 supersymmetry, leading to $\mathrm{N}=3$, rather than $\mathrm{N}=4$, supersymmetry on the $\mathrm{D}=3$ intersection. These configurations are therefore of possible relevance to the dynamics of $\mathrm{D}=3$ gauge theories with $\mathrm{N}=3$ supersymmetry [8].

Given a solution representing a 3-brane intersecting IIB 5-branes, we can Tdualize along a direction in the 2-brane intersection to arrive at a new IIA configuration which can then be lifted back to $D=11$. The resulting solution of $D=11$ supergravity, which generically preserves $3 / 16$ supersymmetry, can be interpreted as an M-theory membrane intersecting, on a string, any number of M-5-branes at arbitrary angles. The special case in which just two M-5-branes intersect orthogonally is itself a new solution ${ }^{\dagger}$, preserving $1 / 4$ of the vacuum supersymmetry, that generalizes the 'two M-5-branes overlapping on a string' solution of [10].

As a further example of how hyper-Kähler manifolds lead via duality to new intersecting branes we consider a 'generalized overlapping fivebrane' solution of $\mathrm{D}=11$ supergravity of the form

$$
\begin{aligned}
d s_{11}^{2} & =\left(H_{1} H_{2}\right)^{\frac{2}{3}}\left[\left(H_{1} H_{2}\right)^{-1} d s^{2}\left(\mathbb{E}^{1,1}\right)+H_{1}^{-1} d s_{4}^{2}\left(M_{2}\right)+H_{2}^{-1} d s_{4}^{2}\left(M_{1}\right)+d z^{2}\right] \\
F & =\left[*_{1} d H_{1}+*_{2} d H_{2}\right] \wedge d z
\end{aligned}
$$

$\dagger$ This solution has been found independently by Tseytlin [9]. 
where $H_{1}$ and $H_{2}$ are harmonic functions on the respective asymptotically-flat hyper-Kähler 4-manifolds $M_{1}$ and $M_{2}$, each with $U(1)$ isometry, and $*_{i}$ indicates the Hodge dual on $M_{i}$. The 'two M-5-branes overlapping on a string' solution is now recovered as the special case for which $M_{1}$ and $M_{2}$ are both chosen to be $\mathbb{E}^{4}$. If one or both $M_{1}$ and $M_{2}$ are taken to be Euclidean Taub-Nut (for example) then $1 / 8$ of the supersymmetry is preserved for appropriate relative signs of the 5-brane charges. Since this 'generalized overlapping fivebrane' solution still has a triholomorphic $T^{2}$ isometry it can be mapped to a solution of IIB supergravity preserving the same fraction of supersymmetry. We show that it maps to a configuration of orthogonally intersecting D-5-branes and NS-5-branes. It can then be mapped back to $D=11$ to yield a new ${ }^{\ddagger} 1 / 8$ supersymmetric solution of $\mathrm{D}=11$ supergravity representing (in the simplest case) four orthogonally intersecting M-5-branes. Remarkably, this can be further generalized to include a pair of intersecting membranes, still preserving $1 / 8$ supersymmetry.

We have been using the terms 'intersecting' and 'overlapping' interchangeably in the above discussion, but there is of course a distinction between them. The possibility of an 'overlapping' brane interpretation arises whenever the branes are potentially separable in one or more directions. If two branes intersect one expects the intersection to appear as a physical intersection in the worldvolume field theory of each brane; this leads, for instance, to the ' $(p-2)$-brane intersection' rule [12]. The solutions considered here are typically translationally invariant in the one direction that potentially separates different branes, so the issue of whether the branes are actually intersecting or merely overlapping is left unresolved. However, the fact that the 'overlapping' M-theory 5-brane solution of [10] has a generalization in which the common 1-brane is naturally interpreted as the intersection of each 5brane with a membrane makes it also natural to suppose that one is left with a mere overlap when the membrane is removed. In any case, we shall find it convenient to adopt this point of view here in order to avoid confusion between different types of

\footnotetext{
$\ddagger$ For example, it is not included in a recent classification of orthogonally intersecting brane solutions [11].
} 
solution. For example, we shall refer to HW-type configurations of IIB D-5-branes and NS-5-branes without 3-branes as 'overlapping' branes whereas we shall refer to the more general configuration with 3-branes as 'intersecting' branes.

\section{Toric Hyper-Kähler manifolds}

We are principally interested here in 8-dimensional hyper-Kähler manifolds with a tri-holomorphic $T^{2}$ isometry, but we shall consider these as special cases of $4 n$-dimensional hyper-Kähler manifolds $\mathcal{M}_{n}$ with a tri-holomorphic $T^{n}$ isometry. We shall refer to them as toric hyper-Kähler manifolds [13]. Let

$$
X^{i}=\frac{\partial}{\partial \varphi_{i}} \quad(i=1, \ldots, n)
$$

be the $n$ commuting Killing vector fields. They are tri-holomorphic if the triplet of Kähler 2-forms $\Omega$ is $\varphi_{i}$-independent, i.e. if

$$
\mathcal{L}_{i} \Omega=0
$$

where $\mathcal{L}_{i}$ is the Lie derivative with respect to $X^{i}$. The general toric hyper-Kähler $4 n$-metric has the local form

$$
d s^{2}=U_{i j} d \mathbf{x}^{i} \cdot d \mathbf{x}^{j}+U^{i j}\left(d \varphi_{i}+A_{i}\right)\left(d \varphi_{j}+A_{j}\right)
$$

where $U_{i j}$ are the entries of a positive definite symmetric $n \times n$ matrix function $U$ of the $n$ sets of coordinates $\mathbf{x}^{i}=\left\{x_{r}^{i} ; r=1,2,3\right\}$ on each of $n$ copies of $\mathbb{E}^{3}$, and $U^{i j}$ are the entries of $U^{-1}$. The $n 1$-forms $A_{i}$ have the form

$$
A_{i}=d \mathbf{x}^{j} \cdot \omega_{j i}
$$

where $\boldsymbol{\omega}$ is a triplet of $n \times n$ matrix functions ${ }^{\star}$ of the $n$ sets of $\mathbb{E}^{3}$ coordinates. The

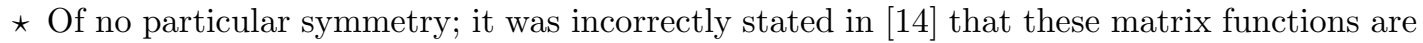
symmetric.
} 
three Kähler 2-forms are [14]

$$
\mathbf{\Omega}=\left(d \varphi_{i}+A_{i}\right) d \mathbf{x}^{i}-\frac{1}{2} U_{i j} d \mathbf{x}^{i} \times d \mathbf{x}^{j}
$$

where $\times$ denotes the standard vector product in $\mathbb{E}^{3}$, the exterior product of forms being understood (e.g. the 3 -component of $d \mathbf{x}^{i} \times d \mathbf{x}^{j}$ is $2 d x_{1}^{i} \wedge d x_{2}^{j}$ ).

For some purposes it is convenient to introduce a (non-coordinate) frame in which the components of both the metric and $\Omega$, and hence the complex structures, are constant. To do so we write $U$ as

$$
U=K^{T} K
$$

for some non-singular matrix $K$ (which is not unique because it may be multiplied on the left by an arbitrary $S O(n)$ matrix). We then define $3 n$ legs of a $4 n$-bein by

$$
\mathbf{E}_{i}=K_{i j} d \mathbf{x}^{j}
$$

and the remaining $n$ legs by

$$
E^{i}=\left(d \phi_{j}+A_{j}\right) K^{j i}
$$

where $K^{i j}$ is the inverse of $K_{i j}$. This $4 n$-bein defines a new frame in which the metric is

$$
d s^{2}=\delta^{i j} E_{i} E_{j}+\frac{1}{2} \delta_{i j} \mathbf{E}^{i} \cdot \mathbf{E}^{j}
$$

and the triplet of Kähler 2-forms is

$$
\Omega=2 E^{i} \mathbf{E}_{i}-\mathbf{E}_{i} \times \mathbf{E}_{j}
$$

In this frame the triplet of complex structures $\mathbf{J}$ are simply a set of three constant $4 n \times 4 n$ matrices 
The conditions on $U_{i j}$ and $A_{i}$ required for the metric to be hyper-Kähler, and for the closure of $\Omega$, are most simply expresed as the constraint [15]

$$
F_{j k i}^{r s}=\varepsilon^{r s t} \partial_{j}^{t} U_{k i}
$$

on the 2-form 'field strengths' $F_{i}=d A_{i}$, for which the components are

$$
F_{j k i}^{r s}=\partial_{j}^{r} \omega_{k i}^{s}-\partial_{k}^{s} \omega_{j i}^{r},
$$

where we have introduced the notation

$$
\frac{\partial}{\partial x_{r}^{i}}=\partial_{i}^{r}
$$

The constraint (2.11) implies that

$$
\partial_{[j}^{t} U_{k] i}=0,
$$

while the 'Bianchi' identity $d F_{i} \equiv 0$ implies that the matrix $U$ satisfies [16]

$$
\partial_{i} \cdot \partial_{j} U=0 \quad(i, j=1, \ldots, n) .
$$

Given a solution of these equations, the 1-forms $A_{i}$ are determined up to a gauge transformation of the form $A_{i} \rightarrow A_{i}+d \alpha_{i}(x)$, which can be compensated by a change of $\varphi_{i}$ coordinates. Thus, the determination of hyper-Kähler metrics of the assumed type reduces to finding solutions of (2.14) and (2.15). We shall refer to these equations as the 'hyper-Kähler conditions'.

Note that (2.15) implies that

$$
U^{i j} \partial_{i} \cdot \partial_{j} U=0
$$

which is equivalent to the statement that each entry of $U$ is a harmonic function on $\mathcal{M}_{n}$. To see that this is so, we observe that the Laplacian, when restricted to 
acting on $T^{n}$-invariant functions, is

$$
\begin{aligned}
\nabla^{2} & =(\operatorname{det} U)^{-1} \partial_{i} \cdot\left(\operatorname{det} U U^{i j} \partial_{j}\right) \\
& =U^{i j} \partial_{i} \cdot \partial_{j}
\end{aligned}
$$

where the second line follows from (2.14). Since $U$ is $T^{n}$-invariant, it follows that (2.16) is equivalent to $\nabla^{2} U=0$. Of course, this is far from being a complete characterization of $U$.

One obvious solution of the hyper-Kähler conditions, which may be considered to represent the 'vacuum', is constant $U$. We shall denote this constant 'vacuum matrix' by $U^{(\infty)}$ (we shall see in due course that this terminology is appropriate for the applications we have in mind). The corresponding 'vacuum metric' is

$$
d s^{2}=U_{i j}^{(\infty)} d \mathbf{x}^{i} \cdot d \mathbf{x}^{j}+U_{(\infty)}^{i j} d \varphi_{i} d \varphi_{j}
$$

For our applications we shall insist that $\varphi_{i}$ be periodically identified with the standard identification

$$
\varphi_{i} \sim \varphi_{i}+2 \pi \quad(i=1, \ldots, n) .
$$

Thus, the 'vacuum manifold' is $\mathbb{E}^{3 n} \times T^{n}$. We shall wish to consider only those hyper-Kähler manifolds that are asymptotic to $\mathbb{E}^{3 n} \times T^{n}$, with the above metric and identifications, as $\left|\mathbf{x}^{i}\right| \rightarrow \infty$ for all $i$. Thus, the moduli space of 'vacua' may be identified with the set of flat metrics on $T^{n}$. This in turn may be identified with the double coset space

$$
S l(n ; \mathbb{Z}) \backslash G l(n ; \mathbb{R}) / S O(n) .
$$

Non-vacuum hyper-Kähler metrics in this class can be found by superposing the constant solution $U^{(\infty)}$ of the hyper-Kähler conditions with some linear com- 
bination of solutions of the form

$$
U_{i j}[\{p\}, \mathbf{a}]=\frac{p_{i} p_{j}}{2\left|\sum_{k} p_{k} \mathbf{x}^{k}-\mathbf{a}\right|}
$$

where $\left\{p_{i}, i=1, \ldots, n\right\}$ is a set of $n$ real numbers and $\mathbf{a}$ is an arbitrary 3 -vector. Any solution of this form may be associated with a $3(n-1)$-plane in $\mathbb{E}^{3 n}$, specified by the 3 -vector equation

$$
\sum_{k=1}^{n} p_{k} \mathbf{x}^{k}=\mathbf{a} .
$$

The associated hyper-Kähler $4 n$-metric is non-singular provided that the parameters $\{p\}$ are coprime integers. We shall henceforth assume that $\{p\}$ denotes an ordered set of coprime integers and we shall refer to this set as a 'p-vector'. The general non-singular metric may now be found by linear superposition. For a given p-vector we may superpose any finite number $N(\{p\})$ of solutions with various distinct 3-vectors $\left\{\mathbf{a}_{m}(\{p\}) ; m=1, \ldots, N\right\}$. We may then superpose any finite number of such solutions. This construction yields a solution of the hyper-Kähler conditions of the form

$$
U_{i j}=U_{i j}^{(\infty)}+\sum_{\{p\}} \sum_{m=1}^{N(\{p\})} U_{i j}\left[\{p\}, \mathbf{a}_{m}(\{p\})\right]
$$

Since each term in the sum is associated to a $3(n-1)$-plane in $\mathbb{E}^{3 n}$, any given solution is specified by the angles and distances between some finite number of mutually intersecting $3(n-1)$-planes [17]. It can be shown that the resulting hyper-Kähler $4 n$-metric is complete provided that no two intersection points, and no two planes, coincide. We demonstrate this in an appendix by means of the hyper-Kähler quotient construction [16].

A feature of this class of hyper-Kähler $4 n$-metrics is that it is $S l(n ; \mathbb{Z})$ invariant, in the sense that, given $S \in S l(n ; \mathbb{Z})$, the $S l(n ; \mathbb{Z})$ transformation $U \rightarrow S^{T} U S$ takes any solution of the hyper-Kähler conditions of the form (2.23) into another 
one of this form. This would be true for $S \in S l(n ; \mathbb{R})$ if the allowed p-vectors were arbitrary, but the restrictions imposed on them by completeness of the metric restricts $S$ to lie in the discrete $S l(n ; \mathbb{Z})$ subgroup. To see this we note that a $3(n-1)$-plane defined by the p-vector $\{p\}$ is transformed into one defined by the new p-vector $S\{p\}$ whose components are again coprime integers only if $S \in$ $S l(n ; \mathbb{Z})$.

It will prove convenient to have some simple examples of complete toric hyperKähler $4 n$-metrics. The simplest examples are found by supposing $\Delta U \equiv U-U^{(\infty)}$ to be diagonal. For example,

$$
U_{i j}=U_{i j}^{(\infty)}+\delta_{i j} \frac{1}{2\left|\mathbf{x}^{i}\right|}
$$

Hyper-Kähler metrics with $U$ of this form were found previously on the moduli space of $n$ distinct fundamental BPS monopoles in maximally-broken rank $(n+1)$ gauge theories [18] (see also [19]). For this reason we shall refer to them as 'LWY metrics'. For the special case in which not only $\Delta U$ but also $U^{(\infty)}$ is diagonal then $U$ is diagonal and the LWY metrics reduce to the metric product of $n$ Euclidean Taub-Nut metrics. There is also a straightforward 'multi-centre' generalization of the LWY metrics which reduces when $U^{(\infty)}$ is diagonal to the metric product of $n$ cyclic ALF spaces (see e.g. [20]). Whenever $\Delta U$ is diagonal we may choose $\boldsymbol{\omega}$ of (2.4) such that $A_{i}$ is a 1 -form on the $i$ th Euclidean 3 -space satisfying

$$
F_{i}=\star d U_{i i} \quad(i=1, \ldots, n)
$$

where $\star$ is the Hodge dual on $\mathbb{E}^{3}$.

For our applications we shall also need to know something about covariantly constant spinors on hyper-Kähler manifolds. We first note that if the holonomy of a $4 n$-dimensional hyper-Kähler manifold is strictly $S p(n)$ (rather than a proper subgroup of it) then there there exist precisely $(n+1)$ covariantly constant $S O(4 n)$ spinors [21]. As we shall explain in more detail in the following section for $n=2$, 
these spinors arise as singlets in the decomposition of the spinor representation of $S O(4 n)$ into representations of $S p(n)$. It will be important in our applications for these covariantly constant spinors to be independent of the $T^{n}$ coordinates. Fortunately this is a consequence of the triholomorphicity of the $T^{n}$ Killing vector fields. This can be seen as follows. Because $\mathrm{X}$ is Killing, its covariant derivative $\nabla X$ is an antisymmetric $4 n \times 4 n$ matrix, i.e. it takes values in the Lie algebra $s o(4 n)$. Let $\Psi$ be a field transforming according to a representation $R$ of $S O(4 n)$ and let $R(\nabla X)$ be the representative of $\nabla X$ in the corresponding representation of $s o(4 n)$. The Lie derivative of $\Psi$ along $X$ is then

$$
\mathcal{L}_{X} \Psi=i_{X} \nabla \Psi+R(\nabla X) \Psi
$$

For a covariantly constant spinor $\eta$ we therefore have that

$$
\mathcal{L}_{X} \eta=\frac{1}{4} \Gamma^{a b}(\nabla X)_{a b} \eta
$$

The condition that $X$ be triholomorphic, when combined with the covariant constancy of the complex structures $\mathbf{J}$, implies that

$$
[\nabla X, \mathbf{J}]=0
$$

This implies that $\nabla X$ actually takes values in the $s p(n)$ subalgebra of $s o(4 n)$, but covariantly constant spinors are $S p(n)$ singlets, so the right hand side of $(2.27)$ vanishes and we deduce that $\mathcal{L}_{X} \eta=0$, as claimed.

Only the $n=2$ cases of the above class of hyper-Kähler manifolds will be needed in our applications. Moreover, for these applications we may restrict $U^{(\infty)}$ to be such that

$$
\operatorname{det} U^{(\infty)}=1
$$

so that the moduli space of 'vacua' is

$$
S l(2 ; \mathbb{Z}) \backslash S l(2 ; \mathbb{R}) / S O(2)
$$

Let us first consider the case in which the metric is determined by just two inter- 
secting 3-planes with p-vectors $\{p\}$ and $\left\{p^{\prime}\right\}$. We can choose the intersection point to be at the origin of $\mathbb{E}^{6}$, so $U$ is given by $U=U^{(\infty)}+\Delta U$, where

$$
2 \Delta U_{i j}=\frac{p_{i} p_{j}}{\left|p_{1} \mathbf{x}^{1}+p_{2} \mathbf{x}^{2}\right|}+\frac{p_{i}^{\prime} p_{j}^{\prime}}{\left|p_{1}^{\prime} \mathbf{x}^{1}+p_{2}^{\prime} \mathbf{x}^{2}\right|}
$$

The orientation of two 3 -planes in $\mathbb{E}^{6}$ is specified by the $3 \times 3$ matrix

$$
M^{r s}=\left(U^{(\infty)}\right)^{i j} \mathbf{n}_{i}^{(r)} \cdot \mathbf{m}_{j}^{(s)}
$$

where $\left\{\mathbf{n}_{i}^{(r)} ; r=1,2,3\right\}$ are three linearly independent unit normals to one 3-plane and $\left\{\mathbf{m}_{i}^{(s)} ; s=1,2,3\right\}$ are three linearly independent unit normals to the other one. The choice of each set of three unit normals is irrelevant, so we are free to choose them in such a way that $M$ is diagonal. Thus, the relative configuration of the two 3-planes is specified, in principle, by three angles. In our case, however, $M$ is $S O(3)$ invariant as a consequence of the $S O(3)$ invariance of the conditions specifying each 3-plane, so

$$
M^{r s}=(\cos \theta) \delta^{r s}
$$

where

$$
\cos \theta=\frac{p \cdot p^{\prime}}{\sqrt{p^{2} p^{\prime 2}}}
$$

with inner product

$$
p \cdot q=\left(U^{(\infty)}\right)^{i j} p_{i} q_{j}
$$

When $p=(1,0)$ and $q=(0,1)$, as is the case for the LWY metrics, (2.34) reduces to

$$
\cos \theta=-\frac{U_{12}^{(\infty)}}{\sqrt{U_{11}^{(\infty)} U_{22}^{(\infty)}}}
$$

Observe that the formula (2.34) for the angle between two 3-planes is $S l(2 ; \mathbb{R})$ invariant, so given any particular two-plane solution we could always choose to 
evaluate the angle between them by making an $S l(2 ; \mathbb{R})$ transformation of coordinates to bring $U^{\infty}$ to the identity matrix. In such coordinates $U$ is diagonal, and the metric is therefore the direct product of two 4-metrics, whenever the two 3-planes are orthogonal. Thus, orthogonality of the two 3-planes implies a reduction of the holonomy from $S p(2)$ to $S p(1) \times S p(1)$. Non-orthogonality of the two 3-planes does not so obviously imply that the holonomy is $S p(2)$ but we have verified, by computation of the curvature tensor, that the holonomy of the LWY metrics is not contained in $S p(1) \times S p(1)$, and so must be $S p(2)$, whenever $U^{\infty}$ is non-diagonal. This is sufficient to show that a metric corresponding to two nonorthogonal 3-planes has $S p(2)$ holonomy. Since the holonomy cannot be reduced by the inclusion of additional 3-planes, a solution of the hyper-Kähler conditions that includes any two non-orthogonal 3-planes yields a metric which also has holonomy $S p(2)$. Thus, the only toric hyper-Kähler 8-metrics for which the holonomy is a proper subgroup of $S p(2)$ are those corresponding to the orthogonal intersection of two 3-planes, or two sets of parallel 3-planes, in which case the metric is the product of two hyper-Kähler 4-metrics.

Finally, we note that for $n=2$ the three Kähler 2-forms can be expressed simply in terms of the three covariantly constant real chiral $S O(8)$ spinors $\eta_{r}$. This is most straightforward in the frame in which these spinors are constant. If we normalize the spinors such that

$$
\left(\eta_{r}\right)^{T} \eta_{r}=1 \quad(r=1,2,3)
$$

then

$$
\Omega_{a b}^{r}=\frac{1}{2} \varepsilon^{r s t}\left(\eta_{s}\right)^{T} \gamma_{a b} \eta_{t}
$$

where $\gamma_{a b}$ is the antisymmetrized product of pairs of $S O(8)$ Dirac matrices. For $n>2$ the relation between the covariantly constant spinors and the triplet of Kähler 2-forms is more involved. We refer the reader to [21] for details. 


\section{Overlapping branes from hyper-Kähler manifolds}

We shall consider first the solution of $\mathrm{D}=11$ supergravity for which the 4 -form field strength vanishes and the 11-metric is

$$
d s_{11}^{2}=d s^{2}\left(\mathbb{E}^{2,1}\right)+U_{i j} d \mathbf{x}^{i} \cdot d \mathbf{x}^{j}+U^{i j}\left(d \varphi_{i}+A_{i}\right)\left(d \varphi_{j}+A_{j}\right)
$$

where $U_{i j}$ is a $2 \times 2$ symmetric matrix of the form (2.23) characterizing an 8dimensional toric hyper-Kähler manifold $\mathcal{M}$. Our first task is to determine the number of supersymmetries preserved by this solution. This is essentially an application of the methods used previously in the context of KK compactifications of $\mathrm{D}=11$ supergravity (see, for example, [26]).

A 32-component real spinor of the $\mathrm{D}=11$ Lorentz group has the following decomposition into representations of $S l(2 ; \mathbb{R}) \times S O(8)$ :

$$
32 \rightarrow\left(2,8_{s}\right) \oplus\left(2,8_{c}\right)
$$

The two different 8-component spinors of $S O(8)$ correspond to the two possible $S O(8)$ chiralities. The unbroken supersymmetries correspond to singlets in the decomposition of the above $S O(8)$ representations with respect to the holonomy group $\mathcal{H}$ of $\mathcal{M}$. For example, the $\mathrm{D}=11$ vacuum corresponds to the choice $U=$ $U^{(\infty)}$ for which $\mathcal{H}$ is trivial; in this case both 8-dimensional spinor representations decompose into 8 singlets, so that all supersymmetries are preserved. The generic holonomy group is $S p(2)$, for which we have the following decomposition of the $S O(8)$ spinor representations:

$$
\begin{aligned}
& \mathbf{8}_{s} \rightarrow \mathbf{5} \oplus \mathbf{1} \oplus \mathbf{1} \oplus \mathbf{1} \\
& \mathbf{8}_{c} \rightarrow \mathbf{4} \oplus \mathbf{4}
\end{aligned}
$$

There are now a total of 6 singlets (three $S l(2 ; \mathbb{R})$ doublets) instead of 32 , so that the $\mathrm{D}=11$ supergravity solution preserves $3 / 16$ of the supersymmetry, unless the 
holonomy happens to be a proper subgroup of $S p(2)$ in which case the above representations must be further decomposed. For example, the $\mathbf{5}$ and $\mathbf{4}$ representations of $S p(2)$ have the decomposition

$$
\begin{aligned}
& 5 \rightarrow(2,2) \oplus(1,1) \\
& 4 \rightarrow(2,1) \oplus(1,2)
\end{aligned}
$$

into representations of $S p(1) \times S p(1)$. We see in this case that there are two more singlets (one $S l(2 ; \mathbb{R})$ doublet), from which it follows that the solution preserves $1 / 4$ of the supersymmetry whenever the holonomy is $S p(1) \times S p(1)$.

The solution (3.1) of $\mathrm{D}=11$ supergravity has no obvious interpretation as it stands, but we shall see how it acquires two distinct interpretations as overlapping or intersecting 5-branes in the context of $\mathrm{D}=10$ IIB supergravity. One such solution involves only D-5-branes and will be discussed in the following section. Here we present a IIB solution involving both Dirichlet $(R \otimes R)$ and Solitonic $(N S \otimes N S)$ 5brane charges. Given that the $\mathrm{D}=11$ fields are invariant under the transformations generated by a $U(1)$ Killing vector field $\partial / \partial y$, the $\mathrm{D}=11$ supergravity action can be reduced to the $\mathrm{D}=10$ IIA supergravity action. The KK ansatz for the bosonic fields leading to the string-frame 10-metric is

$$
\begin{aligned}
d s_{(11)}^{2} & =e^{-\frac{2}{3} \phi(x)} d x^{\mu} d x^{\nu} g_{\mu \nu}(x)+e^{\frac{4}{3} \phi(x)}\left(d y+d x^{\mu} C_{\mu}(x)\right)^{2} \\
A_{(11)} & =A(x)+B(x) \wedge d y
\end{aligned}
$$

where $A_{(11)}$ is the $\mathrm{D}=113$-form potential and $x^{\mu}$ are the $\mathrm{D}=10$ spacetime coordinates. We read off from the right hand side the bosonic fields of $\mathrm{D}=10$ IIA supergravity; these are the $N S \otimes N S$ fields $\left(\phi, g_{\mu \nu}, B_{\mu \nu}\right)$ and the $R \otimes R$ fields $\left(C_{\mu}, A_{\mu \nu \rho}\right)$. In our case we may choose $y=\varphi^{2}$ to arrive at the IIA supergravity 
solution for which the non-vanishing fields are

$$
\begin{aligned}
d s_{10}^{2} & =\left(\frac{U_{11}}{\operatorname{det} U}\right)^{\frac{1}{2}}\left[d s^{2}\left(\mathbb{E}^{2,1}\right)+U_{i j} d \mathbf{x}^{i} \cdot d \mathbf{x}^{j}\right]+\left(\frac{1}{U_{11} \operatorname{det} U}\right)^{\frac{1}{2}}\left(d z+A_{1}\right)^{2} \\
\phi & =\frac{3}{4} \log U_{11}-\frac{3}{4} \log \operatorname{det} U \\
C & =A_{2}-\left(\frac{U_{12}}{U_{11}}\right)\left(A_{1}+d z\right),
\end{aligned}
$$

where we have set $\varphi_{1}=z$. Because of the triholomorphicity of $\partial / \partial y$ the Killing spinors are all $y$-independent and therefore survive as Killing spinors of the reduced theory ${ }^{\star}$.

Since $\phi$ is $z$-independent and $C$ satisfies $\mathcal{L}_{k} C=0$, where $k=\partial / \partial z$, which is a $U(1)$ Killing vector field, we may use the T-duality rules of [28] to map (3.6) to a IIB supergravity solution. Again, the triholomorphicity of $k$ ensures that all Killing spinors survive. Let the $\mathrm{D}=10$ spacetime coordinates be

$$
x^{\mu}=\left(x^{m}, z\right) \quad(m=0,1, \ldots, 8)
$$

where $x^{m}$ are $\mathrm{D}=9$ spacetime coordinates and $z$ is the 'duality direction' coordinate. The T-duality rules for the $N S \otimes N S$ fields, mapping string-frame metric to stringframe metric, are

$$
\begin{aligned}
d \tilde{s}^{2} & =\left[g_{m n}-g_{z z}^{-1}\left(g_{m z} g_{n z}-B_{m z} B_{n z}\right)\right] d x^{m} d x^{n}+2 g_{z z}^{-1} B_{z m} d z d x^{m}+g_{z z}^{-1} d z^{2} \\
\tilde{B} & =\frac{1}{2} d x^{m} \wedge d x^{n}\left[B_{m n}+2 g_{z z}^{-1}\left(g_{m z} B_{n z}\right)\right]+g_{z z}^{-1} g_{z m} d z \wedge d x^{m} \\
\tilde{\phi} & =\phi-\frac{1}{2} \log g_{z z}
\end{aligned}
$$

where we indicate the transformed fields by a tilde. These rules may be read as a map either from IIA to IIB or vice-versa. The only T-duality rules for the $R \otimes R$

\footnotetext{
$\star$ A case in which supersymmetry is broken by dimensional reduction because this condition is not satisfied can be found in [27].
} 
fields that we shall need for most of this paper are those that map from IIA to IIB, with the IIA fields restricted by

$$
B=0 \quad i_{k} A=0,
$$

where $i_{k}$ indicates contraction with the Killing vector field $k=\partial / \partial z$. Given this restriction, the T-dual IIB $R \otimes R$ fields are ${ }^{\dagger}$

$$
\begin{aligned}
\ell & =C_{z} \\
B^{\prime} & =\left[C_{m}-\left(g_{z z}\right)^{-1} C_{z} g_{z m}\right] d x^{m} \wedge d z \\
i_{k} D & =A
\end{aligned}
$$

where $\ell$ is the IIB pseudoscalar, $B^{\prime}$ the $R \otimes R$ two-form potential and $D$ is the IIB 4 -form potential. Because of the self-duality of its field strength we need specify only the components $i_{k} D$ of $D$.

The non-vanishing IIB fields resulting from the application of these T-duality rules to the IIA solution (3.6) are

$$
\begin{aligned}
d s_{E}^{2} & =(\operatorname{det} U)^{\frac{3}{4}}\left[(\operatorname{det} U)^{-1} d s^{2}\left(\mathbb{E}^{2,1}\right)+(\operatorname{det} U)^{-1} U_{i j} d \mathbf{x}^{i} \cdot d \mathbf{x}^{j}+d z^{2}\right] \\
B_{(i)} & =A_{i} \wedge d z \\
\tau & =-\frac{U_{12}}{U_{11}}+i \frac{\sqrt{\operatorname{det} U}}{U_{11}}
\end{aligned}
$$

where

$$
\tau \equiv \ell+i e^{-\phi_{B}} \quad B_{(1)} \equiv B \quad B_{(2)} \equiv B^{\prime}
$$

and $d s_{E}^{2}$ is the Einstein-frame metric, related to the IIB string metric by

$$
d s_{E}^{2}=e^{-\frac{1}{2} \phi_{B}} d s_{B}^{2}
$$

The complex scalar field $\tau$ takes values in the upper half complex plane, on

\footnotetext{
$\dagger$ Our choice of field definitions differs slightly from that of [28].
} 
which the group $S l(2 ; \mathbb{R})$ acts naturally by fractional linear transformations of $\tau$ :

$$
\tau \rightarrow \frac{a \tau+b}{c \tau+d}, \quad\left(\begin{array}{cc}
a & b \\
c & d
\end{array}\right) \equiv S \in S l(2 ; \mathbb{R})
$$

The $\tau$ field equations are invariant under this action, and the invariance extends to the full IIB supergravity field equations with $B_{(i)}$ transforming as an $S l(2 ; \mathbb{R})$ doublet while the Einstein-frame metric is $S l(2 ; \mathbb{R})$ invariant; the fermion transformation properties will not be needed here so we omit them. This invariance allows us to find new solutions as $S l(2 ; \mathbb{R})$ transforms of any given solution. To exploit this observation we note that

$$
\frac{U}{\sqrt{\operatorname{det} U}}=\frac{1}{\mathcal{I} m \tau}\left(\begin{array}{cc}
1 & -\mathcal{R} e \tau \\
-\mathcal{R} e \tau & |\tau|^{2}
\end{array}\right)
$$

which shows that an $S l(2 ; \mathbb{R})$ transformation of $\tau$ induces the linear $S l(2 ; \mathbb{R})$ transformation $U \rightarrow\left(S^{-1}\right)^{T} U S^{-1}$. Thus, given a solution in which $U$ is of the form (2.23) we may find another solution of the IIB field equations for which $U \rightarrow S^{T} U S$ where $S \in S l(2 ; \mathbb{R})$. However, not all of these solutions will correspond to nonsingular solutions of $\mathrm{D}=11$ supergravity. In fact, as we saw earlier in the context of $4 n$-dimensional hyper-Kähler manifolds, the $S l(2 ; \mathbb{R})$ transform of a complete toric hyper-Kähler 8-metric is not itself complete unless $S \in S l(2 ; \mathbb{Z})$. Hence, only an $S l(2 ; \mathbb{Z})$ subgroup of the $S l(2 ; \mathbb{R})$ symmetry group of the IIB field equations is available for generating new solutions if we require non-singularity in $D=11$, and the solutions then generated are just particular cases of those we have already considered.

It is known that, unlike IIB supergravity, IIB superstring theory is not $S l(2 ; \mathbb{R})$ invariant, but it is believed that an $S l(2 ; \mathbb{Z})$ subgroup survives as a symmetry of the full non-perturbative theory, which can be viewed as a limit of a $T^{2}$ compactification of M-theory (see [22] for a recent review). We might therefore have made the restriction to $S l(2 ; \mathbb{Z})$ ab initio on the grounds that this is in any case required 
by M-theory. It is notable, however, that this restriction arises independently from the requirement that our singular IIB intersecting brane solutions be derivable from non-singular solutions of $\mathrm{D}=11$ supergravity. This point has been noted previously $[24,25,1]$ for the 'basic' p-brane solutions in $\mathrm{D}=10$; the principle clearly has some validity but it is not entirely clear why because $D=11$ supergravity is itself as much an effective field theory as are the $\mathrm{D}=10$ supergravity theories. It seems that $\mathrm{D}=11$ supergravity incorporates some of those features of M-theory that are responsible for the resolution of singularities.

We now have a class of solutions of IIB supergravity specified by a set of intersecting 3-planes. As we shall now explain these solutions can be interpreted as overlapping 5-branes. We shall start by considering the case in which $U$ is diagonal. In the simplest of these cases the 8-metric is the metric product of two Euclidean Taub-Nut metrics, each of which is determined by a harmonic function with a single pointlike singularity. Let $H_{i}=\left[1+\left(2\left|\mathbf{x}^{i}\right|\right)^{-1}\right]$ be the two harmonic functions; then

$$
U=\left(\begin{array}{cc}
H_{1}\left(\mathbf{x}^{1}\right) & 0 \\
0 & H_{2}\left(\mathbf{x}^{2}\right)
\end{array}\right)
$$

and the corresponding IIB Einstein metric is

$$
\begin{aligned}
d s_{E}^{2}= & \left(H_{1} H_{2}\right)^{\frac{3}{4}}\left[\left(H_{1} H_{2}\right)^{-1} d s^{2}\left(\mathbb{E}^{2,1}\right)+H_{2}^{-1} d \mathbf{x}^{1} \cdot d \mathbf{x}^{1}\right. \\
& \left.+H_{1}^{-1} d \mathbf{x}^{2} \cdot d \mathbf{x}^{2}+d z^{2}\right] .
\end{aligned}
$$

This is of the form generated by the 'harmonic function rule' $[29,10]$ for the orthogonal intersection of two 5-branes on a 2-brane. Specifically, the singularity of $H_{1}$ is the position of an NS-5-brane, delocalized along the $z$-direction, while the singularity of $H_{2}$ is the position of a D-5-brane, again delocalized along the $z$ direction. The two 5-branes overlap on a 2-brane but are otherwise orthogonal.

To see why the singularities of $H_{1}$ are the positions of NS-branes and those of $H_{2}$ the positions of D-branes we may begin by examining the behaviour of the 
complex scalar $\tau$ as we approach each brane while going far away from from the others. For example, near $\mathbf{x}^{2}=\mathbf{0}$ but for $\left|\mathbf{x}^{1}\right| \rightarrow \infty$ we have

$$
U \sim\left(\begin{array}{cc}
1 & 0 \\
0 & \left(2\left|\mathbf{x}^{2}\right|\right)^{-1}
\end{array}\right)
$$

which shows that the IIB string coupling constant $g_{s}=e^{\phi_{B}}$ goes to zero as $\left|\mathbf{x}^{2}\right| \rightarrow 0$. This shows that the 5 -brane at $\mathbf{x}^{2}=\mathbf{0}$ must be one that is visible in weakly coupled string theory, and this is true only of the D-5-brane. In support of this conclusion we observe that $U$ is well-approximated, in the limit just considered, by the solution of the hyper-Kähler conditions associated with a single 3 -plane with p-vector $(0,1)$. The corresponding solution of IIB supergravity has the feature that only the $R \otimes R$ 2-form potential $B_{(2)}$ is non-zero. In contrast, near $\mathbf{x}^{1}=\mathbf{0}$, but for $\left|\mathbf{x}^{2}\right| \rightarrow \infty$, we have

$$
U \sim\left(\begin{array}{cc}
\left(2\left|\mathbf{x}^{1}\right|\right)^{-1} & 0 \\
0 & 1
\end{array}\right)
$$

which shows that $g_{s} \rightarrow \infty$ as $\left|\mathbf{x}^{1}\right| \rightarrow 0$, so the 5 -brane at $\mathbf{x}^{1}=\mathbf{0}$ cannot be a D-5brane. In fact, it must be a NS-5-brane because the solution of the hyper-Kähler conditions associated with a single 3 -plane with p-vector $(1,0)$ has the feature that only the $N S \otimes N S 2$-form potential $B_{(1)}$ is non-zero.

We are now in a position to interpret the general solution with $U^{(\infty)}=1$. A 'single 3-plane solution' of the hyper-Kähler conditions with p-vector $\left(p_{1}, p_{2}\right)$ is associated with a IIB superstring 5 -brane with 5 -brane charge vector $\left(p_{1}, p_{2}\right)$. This follows simply from the observation that the $S l(2 ; \mathbb{Z})$ transformation that takes a D5 -brane into a 5 -brane with charge vector $\left(p_{1}, p_{2}\right)$ also takes the $\mathrm{D}$-5-brane solution into the solution with $\mathrm{p}$-vector $\left(p_{1}, p_{2}\right)$. Only p-vectors with coprime $p_{1}$ and $p_{2}$ can be found this way, but this is precisely the restriction required by non-singularity of the hyper-Kähler 8-metric. Thus, there is a direct correlation between the angle at which any given 5-brane is rotated, relative to a D-5-brane, and its 5-brane 
charge $^{\star}$. An instructive case to consider is the three 5-brane solution involving a D-5-brane and an NS-5-brane, having orthogonal overlap, and one other 5-brane. As the orientation of the third 5-brane is changed from parallel to the D-5-brane to parallel to the NS-5-brane it changes, chameleon-like, from a D-brane to an NS-brane.

The interpretation of the general solution with non-diagonal $U^{(\infty)}$ is less clear. It might seem that the correlation between the 5-brane charges and their orientations is altered when we allow solutions for which $U^{(\infty)}$ is not the identity matrix since the inner product determining the angle between 3-planes, and hence 5-branes, is altered, whereas the the 2-form potentials $B_{(i)}$ that determine the 5brane charges are unchanged. However, it is likely that the 5-brane charges depend on the expectation value of the complex scalar $\tau$ in such a way that the correlation is maintained. For example, the fundamental string charge on a D-string must be proportional to $\langle\ell\rangle$. This can be seen by considering a D-string stretched between two parallel 3-branes; since $\langle\ell\rangle$ is the vacuum angle in the 3-brane's worldvolume, the stretched D-string must appear as a dyon with electric charge proportional to $\langle\ell\rangle$, by the Witten effect. It follows that the D-string must carry the same fraction of fundamental string charge, and Dirac quantization between strings and 5-branes then implies that the NS-5-brane must carry the same fraction of D-5-brane charge. If we start from $U^{(\infty)}=1$ and then vary $U^{(\infty)}$ at fixed string coupling constant such that $\ell$ goes through one period then the D-5-brane charge on a NS-5-brane must change by one unit. If we now make use of the invariance under shifts of $\ell$ by integral numbers of its periods to return to $\langle\ell\rangle=0$ then we also change the NS5 -brane p-vector from $(1,0)$ to $(1,1)$. Since $U^{(\infty)}$ is again the identity matrix the interpretation of the configuration is as given previously; that is, the angle between the branes is given by the charge vectors. For the above process this is equally true at all intermediate values of $U^{(\infty)}$. This encourages us to believe that the correlation between angles and 5-brane charge is maintained for all $U^{(\infty)}$. Given this, the

\footnotetext{
$\star$ Evidently, this is a consequence of the requirement that the configuration preserve at least $3 / 16$ of the supersymmetry.
} 
interpretation of the IIB solution (3.11) in the general case should now be clear. We have an arbitrary number of 5-branes each specified by a 3 -vector giving its distance from the origin and a p-vector which, together with $U^{(\infty)}$, specifies both its orientation and 5-brane charges. More generally, each 5-brane can be replaced by a set of parallel 5 -branes of the same 5 -brane charge. All of these solutions of IIB supergravity preserve $3 / 16$ supersymmetry.

We conclude this section by mapping the IIB solution (3.11) back to $\mathrm{D}=11$ by a different route. When this solution is T-dualized along one of the space directions of $\mathbb{E}^{2,1}$ we obtain a solution of IIA supergravity which can be lifted back to $\mathrm{D}=11$. A different, but equivalent, route to the same $\mathrm{D}=11$ solution is to dimensionally reduce (3.1) along one of the space directions of $\mathbb{E}^{2,1}$ to get the $\mathrm{D}=10$ IIA supergravity solution with constant dilaton, and metric

$$
d s^{2}=d s^{2}\left(\mathbb{E}^{1,1}\right)+U_{i j} d \mathbf{x}^{i} \cdot d \mathbf{x}^{j}+U^{i j}\left(d \varphi_{i}+A_{i}\right)\left(d \varphi_{j}+A_{j}\right),
$$

all other fields vanishing. We may now T-dualize in both of the $\varphi_{i}$ directions to obtain a new IIA solution. Let $\varphi^{i}$ be the coordinates of the torus dual to the one with coordinates $\varphi_{i}$. Since all fields are of $N S \otimes N S$ type we need only the T-duality rules of (3.8), which yield

$$
\begin{aligned}
d s^{2} & =d s^{2}\left(\mathbb{E}^{1,1}\right)+U_{i j} d X^{i} \cdot d X^{j} \\
B & =A_{i} \wedge d \varphi^{i} \\
\phi & =\frac{1}{2} \log \operatorname{det} U
\end{aligned}
$$

where

$$
X^{i}=\left\{\mathbf{x}^{i}, \varphi^{i}\right\} \quad(i=1,2) .
$$

and $d X \cdot d X$ is the flat metric on $\mathbb{E}^{4}$ (but note that $U$ is still $T^{2}$ invariant so there is no dependence on the $\varphi^{i}$ coordinates). This solution represents an arbitrary number of IIA NS-5-branes intersecting on a string, generalizing previous 
orthogonal intersection solutions of this type [23]. We say 'intersecting' here rather than 'overlapping' because in $\mathrm{D}=10$ there is no separation between the branes (although there is in $\mathrm{D}=11$ ). We should emphasize that there is no actual string on this 'intersection'.

This IIA solution can be lifted to the following solution of $\mathrm{D}=11$ supergravity:

$$
\begin{aligned}
d s_{11}^{2} & =(\operatorname{det} U)^{\frac{2}{3}}\left[(\operatorname{det} U)^{-1} d s^{2}\left(\mathbb{E}^{1,1}\right)+(\operatorname{det} U)^{-1} U_{i j} d X^{i} \cdot d X^{j}+d y^{2}\right] \\
F & =F_{i} \wedge d \varphi^{i} \wedge d y
\end{aligned}
$$

When $U$ is diagonal this reduces to

$$
\begin{aligned}
d s_{11}^{2}= & \left(H_{1} H_{2}\right)^{\frac{2}{3}}\left[\left(H_{1} H_{2}\right)^{-1} d s^{2}\left(\mathbb{E}^{1,1}\right)+H_{1}^{-1} d X^{(2)} \cdot d X^{(2)}\right. \\
& \left.+H_{2}^{-1} d X^{(1)} \cdot d X^{(1)}+d y^{2}\right] \\
F= & F_{i} \wedge d \varphi^{i} \wedge d y
\end{aligned}
$$

This is the special case of the $1 / 4$ supersymmetric 'orthogonal M-5-branes overlapping on a string' solution of [10] for which the harmonic functions $H_{i}$ are harmonic on the $i$ th copy of $\mathbb{E}^{3}$, rather than on the $i$ th copy of $\mathbb{E}^{4}$. When only $\Delta U$ is diagonal, i.e. when $U^{(\infty)}$ is not, the two fivebranes are rotated away from orthogonality and an additional $1 / 16$ of the supersymmetry is broken. In the more general case in which $\Delta U$ is non-diagonal the solution can be interpreted as an arbitrary number of 5-branes intersecting at angles determined by the associated p-vectors; these angles are restricted only by the condition that the pairs of integers $p_{i}$ be coprime. An interesting question, which we do not address here is whether these $3 / 16$ supersymmetric solutions can be generalized to allow $U$ to depend on all eight coordinates $\left\{X^{(i)}, i=1,2\right\}$. 


\section{Non-orthogonal D-branes}

Returning to the IIA solution (3.21), we T-dualize in the common string direction to find an identical solution of IIB supergravity which, consequently, still preserves 3/16 supersymmetry. This IIB solution again represents the overlap on a string of $N S \otimes N S$ 5-branes but it may be mapped to a similar configuration involving only $\mathrm{D}$-5-branes by the weak-strong string coupling $Z_{2} \subset S l(2 ; \mathbb{Z})$ duality. In this way we deduce that

$$
\begin{aligned}
d s_{E}^{2} & =(\operatorname{det} U)^{\frac{1}{4}}\left[d s^{2}\left(\mathbb{E}^{1,1}\right)+U_{i j} d X^{i} \cdot d X^{j}\right] \\
B^{\prime} & =A_{i} \wedge d \varphi^{i} \\
\tau & =i \sqrt{\operatorname{det} U}
\end{aligned}
$$

is also solution of IIB supergravity preserving 3/16 supersymmetry. In the simplest case, in which $U$ is of LWY type, this solution represents the intersection on a string of two D-5-branes, with one rotated relative to the other by an angle $\theta$, given by (2.36). Again, we emphasize that by 'string' we mean here only to indicate the dimensionality of the intersection. Since the D-5-branes have this string direction in common, the configuration is determined by the relative orientation of two 4-planes in the 8-dimensional space spanned by both ${ }^{\star}$. Each 4 -plane can be considered as a quaternionic line in the quaternionic plane. A quaternionic line through the origin is specified by a 2 -vector with components $\left(q_{1}, q_{2}\right)$, where

$$
q_{1}=x^{2}+I x^{3}+J x^{4}+K x^{5} \quad q_{2}=x^{6}+I x^{7}+J x^{8}+K x^{9}
$$

and $I, J, K$ are the quaternionic imaginary units. The orientation of this line is specified by a unit quaternionic 2-vector. The relative orientation of a second quaternionic line through the origin is specified by an element $A$ of $U(2 ; \mathbb{H}) \cong$

\footnotetext{
$\star$ Actually two 3-planes in the supergravity solution but this is due to the partial delocalization of the two 5 -branes.
} 
$S p(2)$. The corrresponding Lie algebra is spanned by $2 \times 2$ quaternionic antihermitian matrices. The diagonal antihermitian matrices generate the $S p(1) \cong S U(2)$ rotations about the origin within a given 4-plane. The off-diagonal quaternion contains the four angles specifying the rotation of one 4-plane relative to another in $\mathbb{E}^{8}$. The group element $A$ will commute with quaternionic conjugation only if it is generated by an element of the Lie algebra with real off-diagonal element. In this case

$$
A=\left(\begin{array}{cc}
\cos \theta & \sin \theta \\
-\sin \theta & \cos \theta
\end{array}\right),
$$

which represents a rotation by an angle $\theta$ of the (2345) 4-plane towards the (6789) 4-plane. The $S O(1,9)$ spinor representation of this particular $S O(8)$ rotation is

$$
R(\theta)=\exp \left\{-\frac{1}{2} \theta\left(\Gamma_{26}+\Gamma_{37}+\Gamma_{48}+\Gamma_{59}\right)\right\} .
$$

We are now in a position to make contact with the work of Berkooz, Douglas and Leigh [3]. They considered two intersecting Dirichlet $(\mathrm{p}+\mathrm{q})$-branes with a common q-brane overlap. According to their analysis, each configuration of this type is associated with an element of $S O(2 p)$ describing the rotation of one $(\mathrm{p}+\mathrm{q})$ brane relative to the other in the 2p-dimensional 'relative transverse' space (in the terminology of [12]). The identity element of $S O(2 p)$ corresponds to parallel branes, which preserve $1 / 2$ the supersymmetry. Other elements correspond to rotated branes. The only case considered explicitly in [3] was an $S U(p)$ rotation, but it was noted that the condition for unbroken supersymmetries was analogous to the reduced holonomy condition arising in KK compactifications. Our case corresponds to an $S p(2)$ rotation in $S O(8)$, We shall now verify that this leads to the preservation of $3 / 16$ supersymmetry.

We recall that the covariantly constant IIB chiral spinors $\epsilon^{A}(A=1,2)$ in the background spacetime of a D-5-brane in the (12345) 5-plane, must satisfy

$$
\Gamma_{012345} \epsilon^{1}=\epsilon^{2} .
$$

If the spacetime includes an additional D-5-brane that is rotated into the (16789) 
5-plane by an angle $\theta$, then $\epsilon^{A}$ must also satisfy

$$
R^{-1} \Gamma_{012345} R \epsilon^{1}=\epsilon^{2}
$$

where $R$ is the matrix in (4.4). From the particular form of this matrix we deduce that (4.6) is equivalent to

$$
R(-2 \theta) \Gamma_{012345} \epsilon^{1}=\epsilon^{2}
$$

which, given (4.5) and (4.4), is equivalent to

$$
\exp \left\{\theta\left(\Gamma_{26}+\Gamma_{37}+\Gamma_{48}+\Gamma_{59}\right)\right\} \epsilon^{A}=\epsilon^{A} \quad(A=1,2) .
$$

Thus, we have to determine the number of simultaneous solutions for two chiral spinors $\epsilon^{A}$ of (4.5) and (4.8).

To proceed, we choose the following representation of the $S O(1,9)$ Dirac matrices:

$$
\begin{aligned}
\Gamma_{0}=\gamma_{5} \otimes \gamma_{5} \otimes i \sigma_{2} & \Gamma_{1}=\gamma_{5} \otimes \gamma_{5} \otimes \sigma_{1} \\
\Gamma_{2}=\gamma_{1} \otimes 1 \otimes 1 & \Gamma_{6}=\gamma_{5} \otimes \gamma_{1} \otimes 1 \\
\Gamma_{3}=\gamma_{2} \otimes 1 \otimes 1 & \Gamma_{7}=\gamma_{5} \otimes \gamma_{2} \otimes 1 \\
\Gamma_{4}=\gamma_{3} \otimes 1 \otimes 1 & \Gamma_{8}=\gamma_{5} \otimes \gamma_{3} \otimes 1 \\
\Gamma_{5}=\gamma_{4} \otimes 1 \otimes 1 & \Gamma_{9}=\gamma_{5} \otimes \gamma_{4} \otimes 1
\end{aligned}
$$

where $\gamma_{1}, \ldots, \gamma_{4}$ are the $4 \times 4 S O(4)$ Dirac matrices and $\gamma_{5}$ is their product. This representation is not real but we may choose each $\gamma_{i}$ to be either real or imaginary, with $\gamma_{5}$ real. The condition (4.5) now reads

$$
\left[\gamma_{5} \otimes 1 \otimes \sigma_{3}\right] \epsilon^{1}=\epsilon^{2}
$$

while the chirality condition is

$$
\left[\gamma_{5} \otimes \gamma_{5} \otimes \sigma_{3}\right] \epsilon^{A}=\epsilon^{A} \quad(A=1,2)
$$


Let $\epsilon_{ \pm}^{A}$ be the eigenspinors of $\sigma_{3}$. Then

$$
\epsilon_{+}^{A}=\left(\begin{array}{c}
\eta_{+}^{A} \\
0
\end{array}\right) \quad \epsilon_{-}^{A}=\left(\begin{array}{c}
0 \\
\eta_{-}^{A}
\end{array}\right)
$$

As a consequence of (4.10) and (4.11), the 16-component spinors $\eta_{ \pm}^{A}$ satisfy

$$
\begin{aligned}
& {\left[1 \otimes \gamma_{5}\right] \eta_{ \pm}^{1}=\eta_{ \pm}^{2}} \\
& {\left[\gamma_{5} \otimes 1\right] \eta_{ \pm}^{1}= \pm \eta_{ \pm}^{2}}
\end{aligned}
$$

while (4.8) is now

$$
\left[\prod_{i=1}^{4} \exp \left\{\theta \gamma_{i} \gamma_{5} \otimes \gamma_{i}\right\}\right] \eta_{ \pm}^{A}=\eta_{ \pm}^{A} \quad(A=1,2)
$$

This is equivalent, given (4.13), to

$$
\sin 2 \theta(1+L) \eta_{+}^{A}=0 \quad \sin \theta \eta_{-}^{A}=0
$$

where

$$
L \equiv\left(\gamma_{1} \gamma_{2} \otimes \gamma_{1} \gamma_{2}+\gamma_{1} \gamma_{3} \otimes \gamma_{1} \gamma_{3}+\gamma_{1} \gamma_{4} \otimes \gamma_{1} \gamma_{4}\right)
$$

In arriving at this result we have used the fact that

$$
\left(L^{2} \mp 2 L-3\right) \eta_{ \pm}^{A}=0
$$

which follows from (4.13).

The conditions (4.15) are trivially satisfied when $\theta=0, \pi$, as expected, and yield only $\eta_{-}^{A}=0$ when $\theta= \pm \pi / 2$, implying that the second D-5-brane reduces the supersymmetry by a factor of $1 / 2$. This is again as expected because the second 
D-5-brane is orthogonal to the first one when $\theta= \pm \pi / 2$, and the constraint (4.6) is equivalent to

$$
\Gamma_{016789} \epsilon^{1}=\epsilon^{2}
$$

as expected of a Killing spinor in the background spacetime of a D-5-brane in the (16789) 5-plane. For all other values of $\theta$ we deduce not only that $\eta_{-}^{A}=0$ but also that

$$
(1+L) \eta_{+}^{A}=0 \quad(A=1,2)
$$

Each of the spinors $\eta_{+}^{1}$ and $\eta_{+}^{2}$ is nominally 16 component but the conditions (4.13) imply that each has only four independent components. Now, we see from (4.17) that $L$ has eigenvalues $-1,3$ when acting on spinors $\eta_{+}$. Since $L$ also has vanishing trace it can be brought to the form

$$
L=\operatorname{diag}(-1,-1,-1,3)
$$

when acting on the 4-dimensional vector space spanned by the four independent solutions of the conditions (4.13) for either $\eta_{+}^{1}$ or $\eta_{+}^{2}$. Thus, (4.19) projects out the eigenvector of $L$ with eigenvalue 3 , leaving only 3 of the 4 independent components of $\eta_{+}^{1}$ or $\eta_{+}^{2}$. We thus have a total of 6 Killing spinors, which should be compared to the 32 Killing spinors of the vacuum, i.e. the intersecting D-brane configuration preserves $3 / 16$ supersymmetry when $\sin 2 \theta \neq 0$. 


\section{Intersecting branes from hyper-Kähler manifolds}

We now return to the $\mathrm{D}=11$ solution (3.1), and generalize it to include a membrane, i.e. we now take as our starting point the $\mathrm{D}=11$ supergravity solution

$$
\begin{aligned}
d s^{2} & =H^{-\frac{2}{3}} d s^{2}\left(\mathbb{E}^{2,1}\right)+H^{\frac{1}{3}}\left[U_{i j} d \mathbf{x}^{i} \cdot d \mathbf{x}^{j}+U^{i j}\left(d \varphi_{i}+A_{i}\right)\left(d \varphi_{j}+A_{j}\right)\right] \\
F & = \pm \omega\left(\mathbb{E}^{2,1}\right) \wedge d H^{-1}
\end{aligned}
$$

where $\omega\left(\mathbb{E}^{2,1}\right)$ is the volume form on $\mathbb{E}^{2,1}$. This is still a solution of $\mathrm{D}=11$ supergravity provided that $H$ is a harmonic function on the hyper-Kähler 8-manifold. Point singularites of $H$ are naturally interpreted as the positions of parallel membranes. For our purposes we require $H$ to be independent of the two $\varphi$ coordinates, so singularities of $H$ will correspond to membranes delocalized on $T^{2}$. Such functions satisfy

$$
U^{i j} \partial_{i} \cdot \partial_{j} H=0
$$

Functions of the form

$$
H=H_{1}\left(\mathbf{x}^{1}\right)+H_{2}\left(\mathbf{x}^{2}\right)
$$

solve this equation if the $H_{i}$ are harmonic on $\mathbb{E}^{3}$, but point singularities of $H_{1}$ or $\mathrm{H}_{2}$ would represent membranes that are delocalized in three more directions. We expect that there exist solutions of (5.2) representing localized membranes (apart from the delocalization on $T^{2}$ ), although explicit solutions may be difficult to find. We would not expect the corresponding 'generalized' membrane solutions (5.1) to be non-singular because this is already not the case for the standard membrane solution (corresponding to $U=1$ ) but it seems likely (by comparison with the $U=1$ case) that the point singularities of $H$ will be horizons that are, if not nonsingular, only mildly singular. In any case, we shall investigate the dual versions of the generalized membrane solutions, as done in the previous section for $H=1$. 
Our first task is again to determine the number of unbroken supersymmetries when $H \neq 1$. One finds that the $S O(1,10)$ Killing spinors $\epsilon$ have the form

$$
\epsilon=H^{-\frac{1}{6}}\left(\begin{array}{l}
\zeta^{1} \\
\zeta^{2}
\end{array}\right)
$$

where $\zeta^{1}$ and $\zeta^{2}$ are two 16-component covariantly constant $S O(8)$ spinors on the hyper-Kähler 8-manifold. In addition, $\epsilon$ satisfies

$$
\Gamma_{012} \epsilon= \pm \epsilon
$$

where the sign on the right hand side is the sign of $F$ in (5.1), i.e. the sign of the membrane charge. Since the product of all eleven Dirac matrices is the identity matrix, this constraint implies that $\zeta^{1}$ and $\zeta^{2}$ have a definite $S O(8)$ chirality (the same for both), depending on the sign of the membrane charge. We can now see from (3.3) that all supersymmetries will be broken if the $S O(8)$ chirality projection is onto the $\boldsymbol{8}_{c}$ representation whereas the fraction of unbroken supersymmetry is unchanged by the inclusion of the membrane if the projection is onto the $\boldsymbol{8}_{s}$ representation. Hence, for an appropriate choice of sign of the membrane charge the field configuration (5.1) is again a solution of $D=11$ supergravity with $3 / 16$ supersymmetry.

Proceeding as before we can now convert this $\mathrm{D}=11$ configuration into a solution of IIB supergravity preserving the same fraction of supersymmetry. The result is

$$
\begin{aligned}
d s_{E}^{2} & =(\operatorname{det} U)^{\frac{3}{4}} H^{\frac{1}{2}}\left[H^{-1}(\operatorname{det} U)^{-1} d s^{2}\left(\mathbb{E}^{2,1}\right)+(\operatorname{det} U)^{-1} U_{i j} d \mathbf{x}^{i} \cdot d \mathbf{x}^{j}+H^{-1} d z^{2}\right] \\
B_{(i)} & =A_{i} \wedge d y \\
\tau & =-\frac{U_{12}}{U_{11}}+i \frac{\sqrt{\operatorname{det} U}}{U_{11}} \\
i_{k} D & =\omega\left(\mathbb{E}^{2,1}\right) \wedge d H^{-1}
\end{aligned}
$$

The singularities of $H$ are now to be interpreted as the locations of parallel D-3branes, in agreement with the 'harmonic function rule'. Otherwise, the solution 
has the same interpretation as before except that it is now natural to interpret the 2-brane overlap of the 5-branes as the intersection with the 3-branes, which are therefore 'stretched' between the 5-branes (i.e. along the $z$ direction), as in the configurations considered in [7].

To obtain the corresponding generalization of (3.21) we first dimensionally reduce (5.1) to obtain the following 'generalized string' solution of IIA supergravity:

$$
\begin{aligned}
d s^{2} & =H^{-1} d s^{2}\left(\mathbb{E}^{1,1}\right)+U_{i j} d \mathbf{x}^{i} \cdot d \mathbf{x}^{j}+U^{i j}\left(d \varphi_{i}+A_{i}\right)\left(d \varphi_{j}+A_{j}\right) \\
B & =\omega\left(\mathbb{E}^{1,1}\right) H^{-1} \\
\phi & =-\frac{1}{2} \log H .
\end{aligned}
$$

A double dualization then yields the new IIA solution

$$
\begin{aligned}
d s^{2} & =H^{-1} d s^{2}\left(\mathbb{E}^{1,1}\right)+U_{i j} d X^{i} \cdot d X^{j} \\
B & =A_{i} \wedge d \varphi^{i}+\omega\left(\mathbb{E}^{1,1}\right) H^{-1} \\
\phi & =\frac{1}{2} \log \operatorname{det} U-\frac{1}{2} \log H
\end{aligned}
$$

where $\varphi^{i}$ are the coordinates of the dual torus. As before, this represents the intersection of NS-5-branes on a string but the string is now an actual IIA string, represented by the harmonic function $H$.

This IIA solution can be lifted to $\mathrm{D}=11$ to give the following generalization of $(3.20)$ :

$$
\begin{gathered}
d s_{11}^{2}=H^{\frac{1}{3}}(\operatorname{det} U)^{\frac{2}{3}}\left[H^{-1}(\operatorname{det} U)^{-1} d s^{2}\left(\mathbb{E}^{1,1}\right)\right. \\
\left.+(\operatorname{det} U)^{-1} U_{i j} d X^{i} \cdot d X^{j}+H^{-1} d y^{2}\right] \\
F=\left[F_{i} \wedge d \varphi^{i}+\omega\left(\mathbb{E}^{1,1}\right) \wedge d H^{-1}\right] \wedge d y .
\end{gathered}
$$

When $U$ is of LWY type this solution represents a set of parallel M-2-branes, located at the singularities of $H$, intersecting two (generically non-orthogonal) M-5-branes on a string. More generally, the M-2-branes intersect any number of M-5-branes, at the singularities of $U$, oriented at essentially arbitrary angles. All of these $\mathrm{D}=11$ supergravity solutions preserve, generically, 3/16 of the supersymmetry. 
Returning to (5.8) we may dualize along the string direction to obtain a IIB solution that also represents the intersection of NS-5-branes, but for which the fundamental string is replaced by a pp wave. A $Z_{2}$ strong/weak coupling duality then yields the IIB solution

$$
\begin{aligned}
d s^{2} & =(\operatorname{det} U)^{\frac{1}{4}}\left[d t d \sigma+H d \sigma^{2}+U_{i j} d X^{i} \cdot d X^{j}\right] \\
B^{\prime} & =A_{i} \wedge d \varphi^{i} \\
\tau & =i \sqrt{\operatorname{det} U}
\end{aligned}
$$

which generalizes (4.1), and also preserves $3 / 16$ of the supersymmetry. It represents the intersection on a string of an arbitrary number of D-5-branes. There is no actual string in the intersection but there is now a pp wave in this direction.

The inclusion of a membrane is natural in the context of $\mathrm{D}=11$ backgrounds involving hyper-Kähler 8-metrics because the transverse space to the membrane is 8-dimensional. In contrast, there is no similarly natural way to include a $\mathrm{D}=11 \mathrm{M}$ 5 -brane because its transverse space is 5-dimensional. However, there is a natural way to incorporate M-5-branes in the special case for which the hyper-Kähler 8metric is the product of two hyper-Kähler 4-metrics. In fact, in this case we may naturally incorporate two M-5-branes. Since $U$ is now assumed diagonal, let $U_{11}=U_{1}\left(\mathbf{x}^{1}\right)$ and $U_{22}=U_{2}\left(\mathbf{x}^{2}\right)$, and let $H_{1}\left(\mathbf{x}^{1}\right)$ and $H_{2}\left(\mathbf{x}^{2}\right)$ be two harmonic functions associated with the two M-5-branes. It will be convenient to introduce two gauge potential one-forms $\tilde{A}_{i}$ with field strength two-forms $\tilde{F}_{i}$, having the same relation to the harmonic functions $H_{i}$ as $F_{i}$ does to the harmonic functions $U_{i}$. That is

$$
\begin{aligned}
& d U_{i}=\star F_{i} \\
& d H_{i}=\star \tilde{F}_{i}
\end{aligned}
$$


where $\star$ is the Hodge dual on $\mathbb{E}^{3}$. Then

$$
\begin{aligned}
d s_{11}^{2}= & \left(H_{1} H_{2}\right)^{\frac{2}{3}}\left[\left(H_{1} H_{2}\right)^{-1} d s^{2}\left(\mathbb{E}^{1,1}\right)+H_{1}^{-1}\left[U_{2} d \mathbf{x}^{2} \cdot d \mathbf{x}^{2}+U_{2}^{-1}\left(d \varphi_{2}+A_{2}\right)^{2}\right]\right. \\
& \left.+H_{2}^{-1}\left[U_{1} d \mathbf{x}^{1} \cdot d \mathbf{x}^{1}+U_{1}^{-1}\left(d \varphi_{1}+A_{1}\right)^{2}\right]+d z^{2}\right] \\
F= & {\left[\tilde{F}_{1} \wedge\left(d \varphi_{1}+A_{1}\right)+\tilde{F}_{2} \wedge\left(d \varphi_{2}+A_{2}\right)\right] \wedge d z }
\end{aligned}
$$

solves the $\mathrm{D}=11$ field equations. Note that the Bianchi identity for $F$ is satisfied since

$$
d F=\sum_{i}\left[\tilde{F}_{i} \wedge F_{i}\right] \wedge d z \equiv 0
$$

as a four-form on $\mathbb{E}^{3}$ vanishes identically. When both $U_{1}$ and $U_{2}$ are constant (5.12) reduces to the orthogonal overlap of two M-5-branes on a string, but with the harmonic functions $H_{i}$ restricted to be independent of the angular coordinates $\varphi_{i}$. In the general case, in which all four harmonic functions $U_{i}$ and $H_{i}$ are non constant, this solution preserves $1 / 8$ supersymmetry provided the relative sign of the 5 -brane charges is chosen appropriately. We shall verify this after mapping the solution into a configuration of intersecting branes.

We first map (5.12) to a IIB solution. Since the restrictions (3.9) no longer apply we should use the full T-duality rules [28], but since all intersections are orthogonal the T-dualized solution can also be deduced from the harmonic function rule. The non-zero fields of the resulting IIB configuration are

$$
\begin{aligned}
d s_{E}^{2}= & \left(H_{1} H_{2} U_{1} U_{2}\right)^{\frac{3}{4}}\left[\left(U_{1} U_{2} H_{1} H_{2}\right)^{-1} d s^{2}\left(\mathbb{E}^{1,1}\right)+\left(U_{2} H_{2}\right)^{-1} d \mathbf{x}^{1} \cdot d \mathbf{x}^{1}\right. \\
& \left.\quad+\left(U_{1} H_{1}\right)^{-1} d \mathbf{x}^{2} \cdot d \mathbf{x}^{2}+\left(H_{1} H_{2}\right)^{-1} d z^{2}+\left(U_{1} U_{2}\right)^{-1} d y^{2}\right] \\
B= & A_{1} \wedge d z+\tilde{A}_{2} \wedge d y \\
B^{\prime}= & A_{2} \wedge d z+\tilde{A}_{1} \wedge d y \\
\tau= & i \sqrt{\frac{H_{1} U_{2}}{H_{2} U_{1}}} .
\end{aligned}
$$

This represents two NS-5-branes and two D-5-branes intersecting orthogonally ac- 
cording to the following pattern

$$
\begin{array}{cccccccccc}
N S: & 1 & 2 & 3 & 4 & 5 & & & & \\
N S: & 1 & & & & & 6 & 7 & 8 & 9 \\
D: & 1 & & & & 5 & 6 & 7 & 8 & \\
D: & 1 & 2 & 3 & 4 & & & & & 9
\end{array}
$$

If this IIB solution is now T-dualized along the 1-direction then the resulting IIA configuration can be lifted to $\mathrm{D}=11$ to give a new solution of $\mathrm{D}=11$ supergravity representing the orthogonal intersection of $4 \mathrm{M}-5$-branes according to the pattern

$$
\begin{array}{llllllllllll}
M: & 1 & 2 & 3 & 4 & 5 & & & & & \\
M: & 1 & & & & & 6 & 7 & 8 & 9 & \\
M: & & & & & 5 & 6 & 7 & 8 & & 10 \\
M: & & 2 & 3 & 4 & & & & & 9 & 10
\end{array}
$$

Since the intersections are all orthogonal the explicit form of the solution is determined by the 'harmonic function rule' together with the information about which coordinates each harmonic function depends on. To fully specify the solution we need therefore give only the latter information. This follows directly from the explicit form of the IIB solution (5.14): the harmonic function associated with a given M-5-brane depends on the coordinates that parameterize the 3-brane intersection of the other M-5-branes. For example, the M-5-brane in the (12345) 5-plane is associated to a harmonic function on the (678) 3-plane. The number of supersymmetries preserved may now be determined as follows. The four M-5-branes lead to the following four conditions on Killing spinors $\epsilon$ :

$$
\begin{array}{cc}
\Gamma_{012345} \epsilon=\epsilon & \Gamma_{016789} \epsilon=\epsilon \\
\Gamma_{0567810} \epsilon=\epsilon & \Gamma_{0234910} \epsilon=\epsilon
\end{array}
$$

Any three of these conditions imply the fourth, so the solution preserves $1 / 8 \mathrm{su}-$ persymmetry. Given any three M-5-branes the signs of the 5-brane charges are 
irrelevant, but the sign of the charge of the fourth M-5-brane is then determined by the requirement that no further supersymmetries be broken; if the other sign is chosen all supersymmetries are broken. When translated back to the original $\mathrm{D}=11$ solution (5.12) this requirement restricts the connection of one of the hyper-Kähler 4-metrics to be either self-dual or anti-self dual according to the choice made for the other one and the choice of signs of the 5-brane charges.

When combined with the fact that the product of all 11 Dirac matrices is the identity matrix, the conditions imply

$$
\Gamma_{059} \epsilon=-\epsilon \quad \Gamma_{0110} \epsilon=\epsilon
$$

so it is possible to further include a pair of membranes, still preserving $1 / 8$ supersymmetry, according to the pattern

$\begin{array}{llllllllllll}M: & 1 & 2 & 3 & 4 & 5 & & & & & \\ M: & 1 & & & & & 6 & 7 & 8 & 9 & \\ M: & & & & & 5 & 6 & 7 & 8 & & 10 \\ M: & & 2 & 3 & 4 & & & & & 9 & 10 \\ M: & 1 & & & & & & & & & 10 \\ M: & & & & & 5 & & & & & & \\ M & & & & & & & \end{array}$

What remains to be determined is on which coordinates the harmonic functions associated to the membranes can depend. We shall not pursue this here. At the same level of analysis we note that if we compactify in the 10-direction then we may also include a IIA D-6-brane in the (234678) 6-plane since it also follows from (5.17) that

$$
\Gamma_{0234678} \epsilon=-\epsilon
$$

A subsequent T-dualization in the 1-direction then yields the a configuration of 
IIB branes intersecting according to the pattern

$\begin{array}{cccccccccc}N S: & 1 & 2 & 3 & 4 & 5 & & & & \\ N S: & 1 & & & & & 6 & 7 & 8 & 9 \\ D: & 1 & & & & 5 & 6 & 7 & 8 & \\ D: & 1 & 2 & 3 & 4 & & & & & 9 \\ W: & 1 & & & & & & & & \\ D: & 1 & & & & 5 & & & & 9 \\ D: & 1 & 2 & 3 & 4 & & 6 & 7 & 8 & \end{array}$

where $W$ indicates a pp wave. Remarkably, this configuration preserves $1 / 8$ of the supersymmetry.

\section{Comments}

In this paper we have constructed several new classes of overlapping and intersecting brane solutions of $\mathrm{D}=10$ and $\mathrm{D}=11$ supergravity theories. We have shown that there is a remarkable latitude allowed by supersymmetry in choosing the relative orientations in multiple brane configurations. In the IIB case, for example, the orthogonal overlap on a 2-brane of a set of parallel D-5-branes with a set of parallel NS-5-branes preserves $1 / 4$ of the supersymmetry. This multiple brane configuration can be deformed to one in which each of an arbitrary number of branes of mixed 5-brane charges overlap at essentially arbitrary angles, while preserving 3/16 supersymmetry. We have found similar configurations involving only D-5-branes, but intersecting on a string, and other configurations of multiple intersecting $\mathrm{D}=11$ fivebranes, all with $3 / 16$ supersymmetry. In all such cases the 3/16 supersymmetry derives from the $S p(2)$ holonomy of hyper-Kähler 8-metrics.

In the case of the IIB D-5-branes we have verified by a quaternionic extension of the analysis of [3] that a pair of intersecting D-5-branes preserves 3/16 supersymmetry if their orientations are related by a rotation in an $S p(2)$ subgroup of $S O(8)$ commuting with multiplication by a quaternion. It was remarked in [3] 
that the determination of the fraction of supersymmetry preserved is analogous to the standard holonomy argument in Kaluza-Klein (KK) compactifications. We can now see that, at least in the $S p(2)$ case, that this analogy is exact because the D-brane configuration corresponds to a IIB supergravity solution that is dual to a non-singular $\mathrm{D}=11$ spacetime of $S p(2)$ holonomy. In view of this it would be of interest to consider other subgroups of $S O(8)$. As pointed out in [3], the holonomy analogy would lead one to expect the existence of intersecting D-brane configurations in which one D-brane is rotated relative to another by an $S U(4), G_{2}$ or Spin(7) rotation matrix. If so, there presumably exist corresponding solutions of IIB supergravity preserving $1 / 8,1 / 8$ and $1 / 16$ of the supersymmetry, respectively. These IIB solutions would presumably have M-theory duals, in which case one is led to wonder whether they could be non-singular (and non-compact) $\mathrm{D}=11$ spacetimes of holonomy $S U(4), G_{2}$ or $\operatorname{Spin}(7)$.

In the case in which a 3-brane intersects overlapping IIB 5-branes, the fact that the solution preserves $3 / 16$ of the supersymmetry implies an $\mathrm{N}=3$ supersymmetry of the field theory on the 2-brane intersection. A massless $\mathrm{D}=3$ field theory with $\mathrm{N}=3$ supersymmetry is automatically $\mathrm{N}=4$ supersymmetric, however, so we conclude that the 2-brane intersection cannot have massless fields, i.e. it is not free to move in any direction. This conclusion is consistent with the conclusion reached in [7] that 3-branes stretched between a D-5-brane and an NS-5-brane have no moduli. It would nevertheless be of interest to learn more about these massive $D=3$ field theories on the 2-brane intersections. It seems that the breaking of $\mathrm{N}=4$ to $\mathrm{N}=3$ supersymmetry is associated with gauge field Chern-Simons terms [8]. Such field theories typically have solitons with interesting properties that may have a 'brane within brane' interpretation which it would be instructive to elucidate.

One other notable feature of some of our new solutions is that the intersection is localized within each brane. For example, in the solution of $D=11$ supergravity representing the intersection of a membrane with overlapping fivebranes the membrane may be localized in all directions other than the one separating the fivebranes. Clearly, there is scope for more semi-localized solutions of this form, 
either by further duality transformations of those constructed here or new solutions constructed by similar methods. Consider, for example, a special case of the 'generalized' membrane solution of (5.1) in which the 8-metric $d s_{8}^{2}$ is the product of $\mathbb{E}^{4}$ with a Euclidean Taub-Nut metric. Reduction on the $S^{1}$ isometry of the latter yields a solution of IIA supergravity in which a membrane is localized inside a 6-brane.

Additional possibilities arise when one considers holomorphic cycles. Consider the case of two parallel IIA 6-branes. When one includes the 11 th dimension, the transverse space is a 4-dimensional toric hyper-Kähler manifold with an $S^{1}$-invariant holomorphic minimal 2 -sphere connecting the two 6-branes. An M-5-brane wrapped around this two-cycle would appear in $\mathrm{D}=10$ as the 3 -brane boundary of a IIA 4-brane stretched between two 6-branes. After T-duality this becomes a 2-brane boundary of a 3-brane stretched between two D-5-branes. Thus, holomorphic cycles provide opportunities to generalize the HW-type IIB solutions given here to include additional 3-branes.

A construction was given in [31] of $S^{1}$-invariant two real dimensional holomorphic minimal submanifolds in general 4-dimensional toric hyper-Kähler metrics. The construction may be generalized to $T^{2}$-invariant holomorphic 4-dimensional submanifolds in toric hyper-Kähler 8-manifolds as follows. We start from $\mathbb{E}^{6}=$ $\mathbb{E}^{3} \times \mathbb{E}^{3}$ with coordinates $\left(\mathbf{x}^{1}, \mathbf{x}^{2}\right)$. A given complex structure

$$
I_{\mathbf{n}}=n_{1} I+n_{2} J+n_{3} K
$$

picks out a common direction in both $\mathbb{E}^{3}$ factors. This defines a 2-plane in $\mathbb{E}^{6}$. Acting with the torus $T^{2}$ gives a 4 real dimensional submanifold of the hyperKähler 8-manifold. Using the explicit form for the complex structures given in section 2 one readily sees that $I_{\mathbf{n}}$ rotates the tangent vectors to the sub-manifold into themselves, which implies that it is holomorphic with respect to that complex structure. It then follows from Wirtinger's theorem that it is minimal. 
One may ask whether these 4 real dimensional submanifolds continue to be holomorphic and minimal in the context of our 'generalized membrane' solutions of $\mathrm{D}=11$ supergravity. The case of most interest is that in which an M-5-brane wraps a 4-cycle that is not the product of two 2-cycles. Assuming that the effective M-5-brane Lagrangian is the 6-volume of the worldvolume in the induced metric, and that $\gamma$ is this induced metric when $H=1$, we deduce from the 11-metric of (5.1) that its Dirac-Nambu-Goto (DNG) Lagrangian is

$$
L_{D N G}=\left(H^{-\frac{2}{3}}\right)\left(H^{\frac{1}{3}}\right)^{2} \sqrt{-\operatorname{det} \gamma}=\sqrt{-\operatorname{det} \gamma}
$$

Remarkably, the factors of $H$ cancel so the 4-dimensional submanifold will continue to be minimal. We therefore expect additional possibilities for intersecting brane solutions to arise in this way. Whether they will continue to preserve the 3/16 supersymmetric can likely be deduced by the 'static probe method' of [32].

Finally, given that there exist so many new intersecting brane solutions of supergravity theories it will be of great interest to see what further insights can be obtained from M-theory or superstring theory. For example, string theory methods could be applied to the configurations of non-orthogonal D-5-branes preserving 3/16 supersymmetry to determine the spectrum of BPS states on the string intersection. One might also investigate in string theory the nature of the singularity associated with the coincidence of intersection points of multiple brane configurations.

\section{Appendix: Completeness via the hyper-Kähler quotient construction}

In this appendix we shall sketch the hyper-Kähler quotient construction of the toric hyper-Kähler $4 n$-metrics and use it to demonstrate their completeness. The discussion follows the lines of [30] and some details have been clarified in discussions with R. Goto as part of a longer project on toric hyper-Kähler manifolds to be

published in the future. The connection between toric hyper-Kähler metrics and arrangements of hyperplanes was pointed out in [17]. 
Suppose we are given a $4 N$-dimensional toric hyper-Kähler manifold $\mathcal{M}_{N}$ with metric $d s^{2}=d y^{\sigma} d y^{\tau} g_{\sigma \tau}$ and a triholomorphic $T^{N}$ isometry. To obtain a new $4 n$-dimensional hyper-Kähler metric $\left.\mathcal{M}_{n}(n<N)\right)$ by the hyper-Kähler quotient construction we first choose a $T^{m}$ subgroup $H$ of the triholomorphic $T^{N}$ group. To each generator of $T^{m}$ we may associate a triplet of moment maps $\mu_{r},(r=1,2,3)$ such that the infinitesimal action of this generator on any of the $4 N$ coordinates $y^{\sigma}$ is

$$
\delta y^{\sigma}=t \Omega_{r}^{\sigma \tau} \frac{\partial \mu_{r}}{\partial y^{\tau}} \quad(r=1,2,3)
$$

where $\Omega_{r}^{\sigma \tau}$ are the entries of the inverse of the $r$ th Kähler 2-form $\Omega_{r}$, and $t$ is an infinitesimal parameter. To obtain the metric on $\mathcal{M}_{n}$ we simply impose the constraints

$$
\boldsymbol{\mu}^{(\alpha)}=-\mathbf{a}^{(\alpha)} \quad(\alpha=1, \ldots, m),
$$

for $m$ triplets of constants $\mathbf{a}^{(\alpha)}$, and project orthogonally to the orbits of $T^{m}$. The $4 n+m$ dimensional submanifold consisting of the solutions of the constraints (A.2) is called the intersection of the level sets of the moment maps. The quotient manifold $\mathcal{M}_{n}$ will be complete if the torus group $T^{m}$ acts smoothly and without fixed points on this intersection. Moreover, it will be invariant under the triholomorphic action of the quotient group $T^{n}=T^{N} / T^{m}$.

To illustrate the method we start with the following flat toric hyper-Kähler metric on $\mathbb{H}^{(n+m)}$ :

$$
\begin{aligned}
d s^{2}=U_{i j}^{(\infty)} d \mathbf{x}^{i} \cdot d \mathbf{x}^{j}+\left(U^{(\infty)}\right)^{i j} d \varphi_{i} d \varphi_{j} \\
\quad+\sum_{\alpha=1}^{m}\left[\frac{d \mathbf{r}^{(\alpha)} \cdot d \mathbf{r}^{(\alpha)}}{2\left|\mathbf{r}^{(\alpha)}\right|}+2\left|\mathbf{r}^{(\alpha)}\right|\left(d \phi_{(\alpha)}+A_{(\alpha)}\right)^{2}\right] .
\end{aligned}
$$

This metric admits the triholomorphic $T^{(m+n)}$ action $(\alpha=1, \ldots, m i=1, \ldots, n)$

$$
\begin{aligned}
\phi_{(\alpha)} & \rightarrow \phi_{(\alpha)}+t_{(\alpha)} \\
\varphi_{i} & \rightarrow \varphi_{i}+t_{i}-p_{i}^{(\alpha)} t_{(\alpha)} .
\end{aligned}
$$

We choose the $T^{m}$ subgroup corresponding to $t_{i}=0$. The moment maps associated 
to this subgroup are

$$
\mathbf{r}^{(\alpha)}-\sum_{i=1}^{n} p_{i}^{(\alpha)} \mathbf{x}^{i}
$$

We therefore impose the constraints

$$
\mathbf{r}^{(\alpha)}-\sum_{i=1}^{n} p_{i}^{(\alpha)} \mathbf{x}^{i}+\mathbf{a}^{(\alpha)}=0
$$

for some constants $\mathbf{a}^{(\alpha)}$, which specify the level sets of the moment maps. We must now check that $T^{m}$ acts smoothly and freely on the intersection of these level sets. It is clear that the quantities $p_{i}^{(\alpha)}$ must be rational because otherwise the $T^{m}$ action would be ergodic. If they have common divisor then one may find an element of $T^{m}$ which does not act freely on the intersection of level sets. Finally if two planes coincide, or if more than two planes intersect at a point, then the group $T^{m}$ will have fixed points on the intersection of the level sets and the quotient will again be singular. For a more detailed mathematical discussion of completeness (albeit in a slightly different setting) the reader is referred to [17].

If (A.6) is now used in (A.3) to eliminate the $\mathbf{r}^{(\alpha)}$ in favour of the $\mathbf{x}^{i}$ then we get a new toric hyper-Kähler $4 n$-metric with $U$ of the form (2.23). The quotient space continues to admit a triholomorphic $T^{n}$ action, given by $\varphi_{i} \rightarrow \varphi_{i}+t_{i}$.

Acknowledgements: We thank E. Bergshoeff, R. Goto and A. Tseytlin for informative discussions. G.P. was supported by a Royal Society University Research Fellowship. 


\section{REFERENCES}

1. P.K. Townsend, The eleven-dimensional supermembrane revisited, Phys. Lett. 350B (1995) 184.

2. M. Costa and G. Papadopoulos, Superstring dualities and p-brane bound states, hep-th/9612204.

3. M. Berkooz, M.R. Douglas and R.G. Leigh, Branes Intersecting at Angles, Nucl. Phys. B480 (1996) 265.

4. V. Balasubramanian and R.G. Leigh, D-branes, moduli and supersymmetry, hep-th/9611165.

5. K. Becker and M. Becker, M-theory on eight-manifolds, Nucl. Phys. B477 (1996) 155.

6. M.J. Duff, H. Lü, C.N. Pope and E. Sezgin, Supermembranes with fewer supersymmetries, Phys. Lett. 371B (1996) 206.

7. A. Hanany and E. Witten, Type IIB superstrings, BPS monopoles and three-dimensional gauge theories, hep-th/9611230.

8. B.M. Zupnik and D.V. Khetselius, Three-dimensional extended supersymmetry in harmonic superspace, Yad. Fiz. 47 (1988) 1147; H-C. Kao and K. Lee, Self-dual Chern-Simons Higgs Systems with an $N=3$ extended supersymmetry, Phys. Rev. D46 (1992) 4691; H-C. Kao, Self-dual Yang-Mills Chern-Simons Higgs systems with an $N=3$ extended supersymmetry, Phys. Rev. D50 (1994) 2881.

9. A.A. Tseytlin, Composite BPS configurations of p-branes in 10 and 11 dimensions, hep-th/9702163.

10. J.P. Gauntlett, D.A. Kastor and J. Traschen, Overlapping Branes in MTheory, Nucl. Phys. B478 (1996) 544.

11. E. Bergshoeff, M. de Roo, E. Eyras, B. Janssen and J.P. van der Schaar, Multiple Intersections of D-branes and M-branes, hep-th 9612095. 
12. G. Papadopoulos and P.K. Townsend, Intersecting M-branes, Phys. Lett. 380B (1996) 273.

13. R. Goto, On hyper-Kähler manifolds of type $A_{\infty}$, Geom. Funct. Anal. 4 (4) (1994) 424; On toric hyper-Kähler manifolds, Kyoto Univ. preprint RIMS-818 (1991)

14. G. Papadopoulos and P.K. Townsend, Solitons in supersymmetric sigmamodels with torsion, Nucl. Phys. B444 (1995) 245.

15. H. Pederson and Y-S Poon, Commun. Math. Phys. 117 (1988) 569.

16. N. Hitchin, A. Karlhede, U. Lindström and M. Roček, Commun. Math. Phys. 108 (1987) 537.

17. R. Bilawski and A.S. Dancer, The geometry and topology of toric hyperKähler manifolds, McMaster preprint (1996).

18. K. Lee, E. Weinberg and P. Yi, The moduli space of many BPS monopoles, hep-th/9602167.

19. G.W. Gibbons and N.S. Manton, The moduli space metric for well-separated BPS monopoles, Phys. Lett. 356B (1995) 32.

20. G.W. Gibbons and P.J. Ruback, Commun. Math. Phys. 115 (1988) 267.

21. M.Y. Wang, Parallel spinors and parallel forms, Ann. Global. Anal. Geom. 7 (1989) 59 .

22. P.K. Townsend, Four lectures on M-theory, hep-th/9612121.

23. R.R. Khuri, Remark on string solitons, Phys. Rev. D48 (1993) 2947; N.D. Lambert, Heterotic p-branes from massive sigma models, Nucl. Phys. 477 (1996) 141.

24. M.J. Duff, G.W. Gibbons and P.K. Townsend, Macroscopic superstrings as interpolating solitons, Phys. Lett. 332 B (1994) 321. 
25. G.W. Gibbons, G.T. Horowitz and P.K. Townsend, Higher-dimensional resolution of dilatonic black hole singularities, Class. Quantum Grav. 12 (1995) 297.

26. M.J. Duff, B.E.W. Nilsson and C.N. Pope, Kaluza-Klein Supergravity, Phys. Rep. 130 (1986) 1.

27. H. Lü, C.N. Pope and P.K. Townsend, Domain walls from anti-de Sitter space, Phys. Lett. 391B (1997) 39.

28. E. Bergshoeff, C.M. Hull and T. Ortín, Duality in the type II superstring effective action, Nucl. Phys. B451 (1995) 547.

29. A. Tseytlin, Harmonic superpositions of M-branes, Nucl. Phys. B475 (1996) 149.

30. G.W. Gibbons, P. Rychenkova and R. Goto, Hyper-Kähler quotient construction of BPS monopole moduli spaces, hep-th/9608085.

31. G.W. Gibbons and P.J. Ruback, Winding strings, Kaluza-Klein monopoles and Runge-Lenz vectors, Phys. Lett. 215B (1988) 653.

32. A.A. Tseytlin, No force condition and BPS combinations of p-branes in 11 and 10 dimensions, hep-th/9609212. 